\title{
Strong narrow-band electron cyclotron emission from a mirror plasma heated by electron cyclotron waves
}

$\operatorname{AUTHOR}(\mathrm{S}):$

Ito, K; Kiwamoto, Y; Saito, T; Tatematsu, Y

\section{CITATION:}

Ito, K ... [et al]. Strong narrow-band electron cyclotron emission from a mirror plasma heated by electron cyclotron waves. PHYSICS OF PLASMAS 2000, 7(12): 4923-4930

\section{ISSUE DATE:}

2000-12

URL:

http://hdl.handle.net/2433/49822

\section{RIGHT:}

Copyright 2000 American Institute of Physics. This article may be downloaded for personal use only. Any other use requires prior permission of the author and the American Institute of Physics. 


\title{
Strong narrow-band electron cyclotron emission from a mirror plasma heated by electron cyclotron waves
}

\author{
K. Ito ${ }^{\text {a) }}$ \\ Graduate School of Human and Environmental Studies, Kyoto University, Sakyo-ku, Kyoto 606-8501, Japan \\ Y. Kiwamoto \\ Faculty of Integrated Human Studies, Kyoto University, Sakyo-ku, Kyoto 606-8501, Japan \\ T. Saito and Y. Tatematsu \\ Plasma Research Center, University of Tsukuba, Tsukuba, Ibaraki 305-8577, Japan
}

(Received 21 April 2000; accepted 30 August 2000)

\begin{abstract}
Strong electromagnetic radiation is observed in a narrow band slightly above the frequency of a heating wave that is absorbed by electrons near the electron cyclotron resonance layer in a magnetic beach. The frequency spectrum consists of a sharp component and a broad background, which is enhanced by more than $30 \mathrm{~dB}$ and $10 \mathrm{~dB}$, respectively, above the radiation of thermal electrons. This observation is explained in terms of cyclotron radiation emitted by electrons, localized in a magnetic mirror, that are resonantly heated and bunched by a strong monochromatic wave. (C) 2000 American Institute of Physics. [S1070-664X(00)04712-1]
\end{abstract}

\section{INTRODUCTION}

Electron cyclotron emission (ECE) has been widely utilized for measuring the temperature of electrons in magnetically confined hot plasmas. ${ }^{1-4}$ The frequency spectra of the emission are quite sensitive to the structure of the velocity distributions, ${ }^{1}$ and the application of ECE has been limited to the temperature measurement in fully thermalized plasmas as confined in toroidal traps. This sensitivity of the radiation spectra, however, does also provide a useful means to study microscopic processes in wave-particle interactions. ${ }^{5}$

In this paper we study structures in the frequency spectra of electromagnetic radiation emitted from electrons that are heated by a strong monochromatic wave at the fundamental electron cyclotron resonance layer along a magnetic beach. ${ }^{6,7}$ The experiment is carried out in one of the end mirror cells of a tandem mirror. The plasma is deep in the weakly collisional regime and the scale-length of the magnetic inhomogeneity is much shorter than the collision mean-free-path. Therefore, we expect that the cyclotron emission from the electrons should be quite different from that emanating from Maxwellian electrons, because the velocity distribution bears a microscopic structure that characterizes resonant waveparticle interactions.

There have been many arguments about the electron velocity distribution under strong electron cyclotron resonance heating (ECRH) in a tandem mirror. A substantial deformation of the velocity distribution was called for to explain such observations as a large transmission efficiency of a strong cyclotron wave through the magnetic beach ${ }^{7}$ and the enhanced electron flux along the magnetic field. ${ }^{7-9}$ It was supported by an $\mathrm{x}$-ray analysis of the local electron distribution. ${ }^{10}$ The potential profile that is essential for confinement in a tandem mirror configuration is strongly

${ }^{a)}$ Electronic mail: n50116@ sakura.kudpc.kyoto-u.ac.jp coupled with the shape of the velocity distribution of electrons modified by ECRH. ${ }^{11}$

These discussions, however, have been limited to a timeaveraged velocity distribution. Because the radiation around the cyclotron frequency is connected to the fast motion of electrons interacting with the heating waves, one of expectations with the present experiment was that new features relevant to ECRH in the magnetic beach configuration might be observed. Actually, we have observed a new type of radiation that is highly enhanced over thermal emissions in a narrow band that is clearly separated from but closely located to the heating frequency. In this paper, though we have not reached a complete understanding, we report in some detail the observational facts about this radiation and discuss possible mechanisms for the emission.

The construction of this paper is as follows. The experimental method is given in Sec. II. The experimental results are shown in Sec. III and discussed in Sec. IV. A conclusion is given in Sec. V.

\section{EXPERIMENTAL METHOD}

\section{A. Experimental configuration}

The experiment is carried out with a plasma in the east plug/barrier cell of the GAMMA10 tandem mirror as illustrated schematically in Fig. 1. ${ }^{12-14}$ This cell is an axisymmetric mirror that is connected to the central solenoid through a quadrapole mirror cell along the magnetic field lines. For convenience we take the right-hand coordinate system in which the origin is at the center of the central solenoid, $z$ axis running from the east to the west along the machine axis, and $x$ and $y$ axes pointing upward and southward, respectively. The plug/barrier cell has the minimum on-axis magnetic field of $0.495 \mathrm{~T}$ at the local mid-plane at $z=-8.8 \mathrm{~m}$, and the maximum field of $3 \mathrm{~T}$ at $z=-7.56 \mathrm{~m}$ and $z=-10.04 \mathrm{~m}$. The plasma is produced in the central solenoid by radio- 


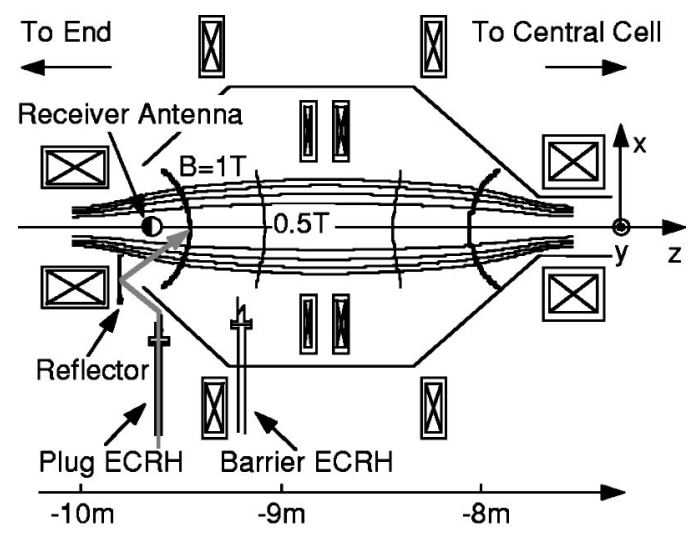

FIG. 1. A schematic view of the east plug/barrier cell of the GAMMA10 tandem mirror.

frequency (RF) wave heating, and is kept from flowing out along the field lines by a potential profile created in the plug/ barrier cells at the machine ends. ${ }^{12,13}$

Electron cyclotron resonance heating (ECRH) is used to produce the potential profile. The ECRH power at $28 \mathrm{GHz}$ is delivered to the fundamental resonance layer of $1 \mathrm{~T}$ for the plug and to the second harmonic layer of $0.5 \mathrm{~T}$ near the mid-plane for the thermal barrier. The heating power, variable up to $150 \mathrm{~kW}$, is generated in a gyrotron, transmitted through an over-moded cylindrical wave-guide system, and converted from the $\mathrm{TE}_{02}$ wave-guide mode to linearly polarized beam with Vlasov-Nakajima antenna., ${ }^{71}$ The microwave beam for the plug electron heating is reflected on a rotatable curved mirror and launched obliquely to the machine axis toward the resonance layer as an X-mode cyclotron wave. The direction of the beam is steered by the reflecting mirror so that the crossing point of the beam through the $1 \mathrm{~T}$ surface moves in the $x$-direction. The total efficiency of the transmission and the conversions is about $60 \%$. The $e^{-1}$ width of the profile of the beam power at the resonance layer is typically $0.10 \mathrm{~m}$ at the plug and $0.15 \mathrm{~m}$ at the barrier. $^{14}$

Before the application of the ECRH, the local electron density is $n_{e} \approx 10^{17} \mathrm{~m}^{-3}$ and the temperature $T_{e} \approx 30-40$ $\mathrm{eV}$. With the ECRH at the plug, $T_{e}$ increases up to around $500 \mathrm{eV}$ and the density decreases by some factor, so that the electrons enter deep into the collisionless regime. In much less than $100 \mu$ s after the start of the ECRH, the potential profile is established, and an enhanced axial flux of warm electrons of several hundreds $\mathrm{eV}$ is detected at the machine end. It indicates an intimate relation between the electron transport in the velocity space caused by the heating and its spatial transport resulting in the formation of potential distribution. ${ }^{7-10,15}$

\section{B. Diagnostic method}

In addition to the standard diagnostic devices, we have set up a new system for receiving and analyzing electromagnetic radiation in the electron cyclotron range of frequencies as illustrated in Fig. 2(a). This system covers two frequency bands. One is in the fundamental resonance range $(28 \mathrm{GHz})$, and another is for the second harmonic range $(56 \mathrm{GHz})$.

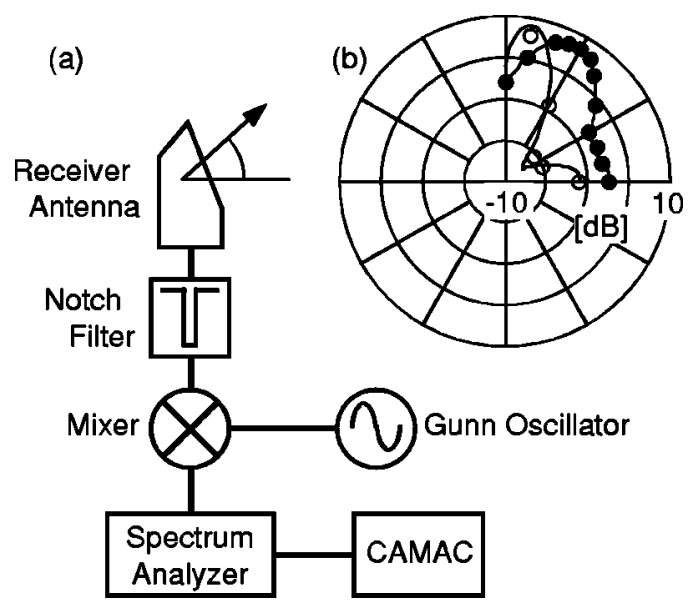

FIG. 2. (a) A schematic diagram of the emission detection system. (b) Directivity of the receiver antenna at $28 \mathrm{GHz}$ (closed circles) and at $56 \mathrm{GHz}$ (open circles).

They are similar in construction, consisting of a movable receiver antenna, a heterodyne mixer with a Gunn oscillator, and a spectrum analyzer.

The microwave emission is collected on the receiver antenna located at $z=-9.77 \mathrm{~m}$ in the east plug adjacent to the region where the electron cyclotron waves used for heating are absorbed. The antenna is used both for the $28 \mathrm{GHz}$ and for the $56 \mathrm{GHz}$ range. It is radially movable from $y=0.25$ to $0.81 \mathrm{~m}$ and can be rotated around the $y$-axis. Its structure is shown schematically in Fig. 2(a). The radiation enters a 16 $\mathrm{mm}$ diam cylindrical wave-guide through a plain aperture with its normal pointing 70 deg to the wave-guide axis. Figure 2(b) shows the gain of the antenna at $28 \mathrm{GHz}$ (closed circles) and $56 \mathrm{GHz}$ (open circles). The gain is defined as a ratio of received powers of an antenna $W_{r}$ to that with nondirectivity antenna $W_{0}$,

$$
G_{r}=\frac{W_{r}}{W_{0}}=\frac{4 \pi^{2}}{\lambda_{o}^{2}} A_{e},
$$

where $\lambda_{o}$ and $A_{e}$ are the free-space wavelength of a microwave and the effective area of the antenna, respectively. The sensitivity at $28 \mathrm{GHz}$ is maximized in the direction of $65 \mathrm{deg}$ to the wave-guide axis, and the $3 \mathrm{~dB}$ width is $30 \mathrm{deg}$. In most cases, we set the receiver antenna at $y=0.275 \mathrm{~m}$ because it looks to the on-axis resonance point with the maximum sensitivity there.

The collected microwave power propagates through the $2 \mathrm{~m}$ long over-sized circular wave-guide and passes through a vacuum-sealed window to be converted to the $\mathrm{TE}_{10}$ mode of a rectangular wave-guide. The rectangular wave-guide for a $28 \mathrm{GHz}$ channel includes a notch filter with a $30 \mathrm{~dB}$ rejection band from 27.95 to $28.10 \mathrm{GHz}$ for protecting sensitive devices from being damaged by the heating power. The signals are converted down to the range below $1.8 \mathrm{GHz}$ through a heterodyne circuit that consists of a local oscillator of Gunn diode and a balanced mixer, and finally fed to a spectrum analyzer for frequency analyses. The spectrum analyzer puts forth voltage signals corresponding to the selected frequency band and the power within the band of $5 \mathrm{MHz}$. These 


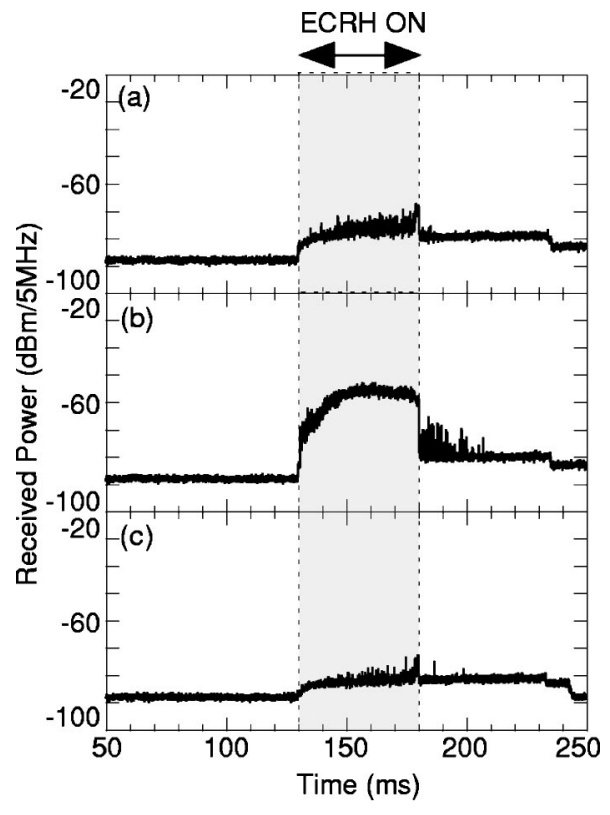

FIG. 3. Time evolution of different frequency components of emission. (a) $f=27.5 \pm 0.2 \mathrm{GHz}$; (b) $f=28.5 \pm 0.2 \mathrm{GHz}$; (c) $f=29.5 \pm 0.2 \mathrm{GHz}$.

signals are recorded on a Computer Automated Measurement And Control (CAMAC) system including Analog-Digital Conversion with a sampling time of $40 \mu \mathrm{s}$.

The electric properties of all components are tested in the whole frequency range of interest so that we can evaluate the absolute value of the collected microwave power. It is confirmed that the total response of the integrated system varies only mildly against frequency, except within the filtered band. The typical transmission efficiency is $\eta_{f}=-20$ $\mathrm{dB}$ in the $28 \mathrm{GHz}$ range and $\eta_{s}=-18 \mathrm{~dB}$ in the $56 \mathrm{GHz}$ range.

\section{EXPERIMENTAL RESULTS}

\section{A. Time evolution of radiation spectra}

We examine how the frequency spectrum of the radiation evolves in time after the start of the ECRH. Figure 3 plots the time evolution of the power of several components in the frequency spectrum. The plasma is produced from 50 to $230 \mathrm{~ms}$, and the plug ECRH is applied from 130 to $180 \mathrm{~ms}$ with a gyrotron power of $100 \mathrm{~kW}$. The analyzer frequency $f_{A}$ is fixed at $0.2 \mathrm{GHz}$ with the resolution band width (RBW) of $5 \mathrm{MHz}$ while the frequency $f_{\mathrm{LO}}$ of the local oscillator fed to the mixer is varied as at $27.5,28.5,29.5 \mathrm{GHz}$, downward. The displayed intensity represents the sum of the components at $f_{\mathrm{LO}}+0.2 \mathrm{GHz}$ and $f_{\mathrm{LO}}-0.2 \mathrm{GHz}$ with width of 5 $\mathrm{MHz}$.

An enhancement of intensity is observed at each frequency component immediately after the application of the ECRH. The enhancement around 27.5 and $29.5 \mathrm{GHz}$ is by several $\mathrm{dB}$ above the level detected before the ECRH. On the other hand, we observe a substantial enhancement of more than $30 \mathrm{~dB}$ around $28.5 \mathrm{GHz}$. The signal level of each frequency component does not change appreciably during the period of the ECRH. After the ECRH is turned off, these

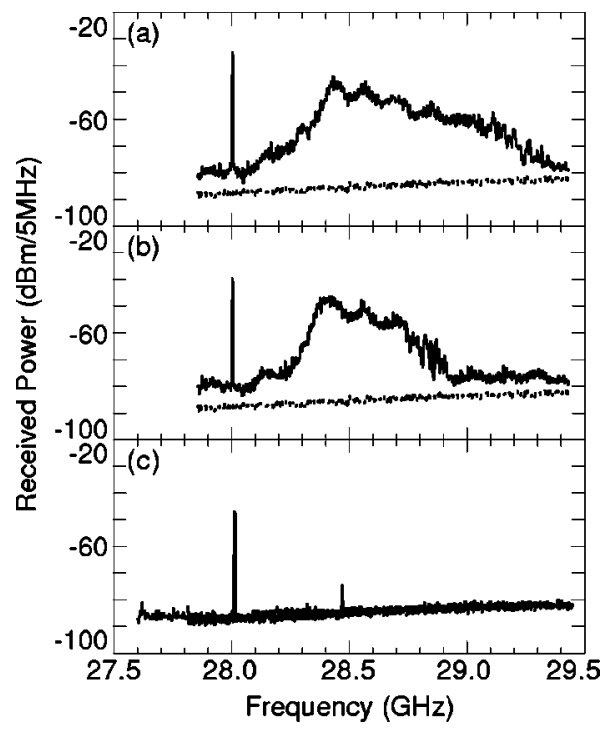

FIG. 4. Frequency spectra associated with the following different heating combinations: (a) with a full set of ECRH, (b) with the east plug ECRH, (c) with the west plug ECRH or the barrier ECRH. The dotted lines represent the noise level of the diagnostic system.

signals go down but still remain observed until the plasma disappears. The amplitude of the post-ECRH radiation is almost $10 \mathrm{~dB}$ above that before the ECRH.

By experimentally confirming that the frequency spectrum of the radiation does not change appreciably during the $\mathrm{ECRH}$, we obtain the spectrum in a wide range in one shot by continuously sweeping $f_{A}$ from 0 to $1.8 \mathrm{GHz}$ for $20 \mathrm{~ms}$. Since upper and lower sideband signals are not discriminated, the analyzed spectrum ranges from $f_{\mathrm{LO}}-1.8 \mathrm{GHz}$ to $f_{\mathrm{LO}}+1.8 \mathrm{GHz}$. For discrimination we vary $f_{\mathrm{LO}}$ and compare the resultant spectra. From shifts of each frequency component in the synthesized spectra, we can determine the correct frequency of the observed radiation.

\section{B. Narrow-band radiation}

There are four channels of ECRH that are usually applied simultaneously to establish the tandem mirror potential configuration. To evaluate the relative significance of the ECRH channels in the contribution to the enhanced radiation, the frequency spectra associated with different combinations of ECRH are examined as shown in Fig. 4. In each plate, the dotted line represents the noise level of this system as obtained in vacuum shots without the plasma. The power emitted from cold electrons before ECRH is lower than this noise level.

Figure 4(a) shows the observed spectrum when a full set of ECRH is applied with each gyrotron power of $140 \mathrm{~kW}$. An enhancement by several $\mathrm{dB}$ is observed over a wide frequency range around the fundamental resonance (26-30 $\mathrm{GHz}$ ). In addition, we notice a substantial enhancement of more than $30 \mathrm{~dB}$ around $28.4 \mathrm{GHz}$. The $10 \mathrm{~dB}$ frequency width of this component is quite narrow (about $0.4 \mathrm{GHz}$ ). The sharp spike at $28 \mathrm{GHz}$ corresponds to a stray field of the heating wave. 


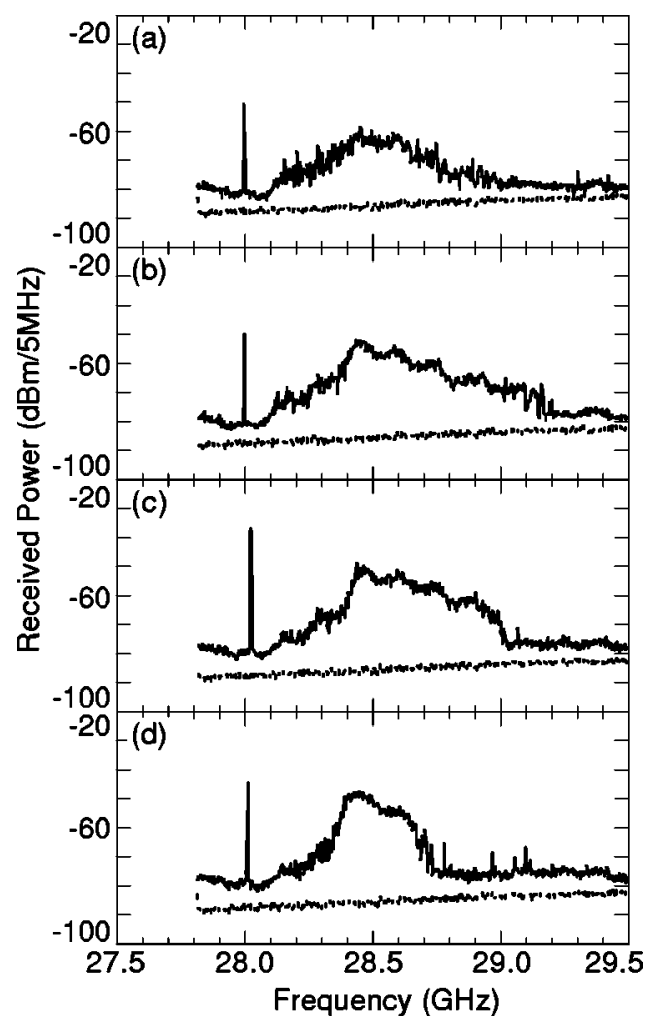

FIG. 5. Frequency spectra at different levels of the plug ECRH power: (a) 20, (b) 60, (c) 100, , (d) $140 \mathrm{~kW}$.

Figure 4(b) shows the spectrum when only the east plug ECRH is applied with the gyrotron power of $140 \mathrm{~kW}$. The same amount of enhancement is observed, and we notice that statistically there is no difference between the two cases. The spectrum of the radiation when we apply only the barrier ECRH with the gyrotron power of $100 \mathrm{~kW}$ is shown in Fig. 4(c). The spectrum when only the west plug ECRH is at work with the gyrotron power of $140 \mathrm{~kW}$ is also superposed in this plate. In either case, we do not observe any enhancement of the radiation. The small sharp spike at $28.5 \mathrm{GHz}$ is attributed to the unblocked parasitic power generated at the higher-order $\mathrm{TE}_{022}$ resonance of the gyrotron cavity. ${ }^{16}$ From these data, we conclude that the enhanced radiation from the plasma is solely attributed to the east plug ECRH that works at the mirror cell where the measurements are made. This fact indicates that the electrons responsible for the enhanced radiation are created by the fundamental ECRH, and that most of them are localized in the mirror cell under the heating.

The next question to be answered is how the spectrum depends on the power level of the heating wave. Figure 5 shows the frequency spectra observed at different levels of the plug ECRH power such as (a) $20 \mathrm{~kW}$, (b) $60 \mathrm{~kW}$, (c) 100 $\mathrm{kW}$, and (d) $140 \mathrm{~kW}$. In each plate, the dotted line represents the noise level of the diagnostic system. The examination below $20 \mathrm{~kW}$ was not available due to unstable generation of the gyrotron power. One can observe that the level of the broad component extending beyond $29 \mathrm{GHz}$ increases with the heating power. This trend of leveling-up is confirmed experimentally over the wide spectral range of $27.8-29.6$

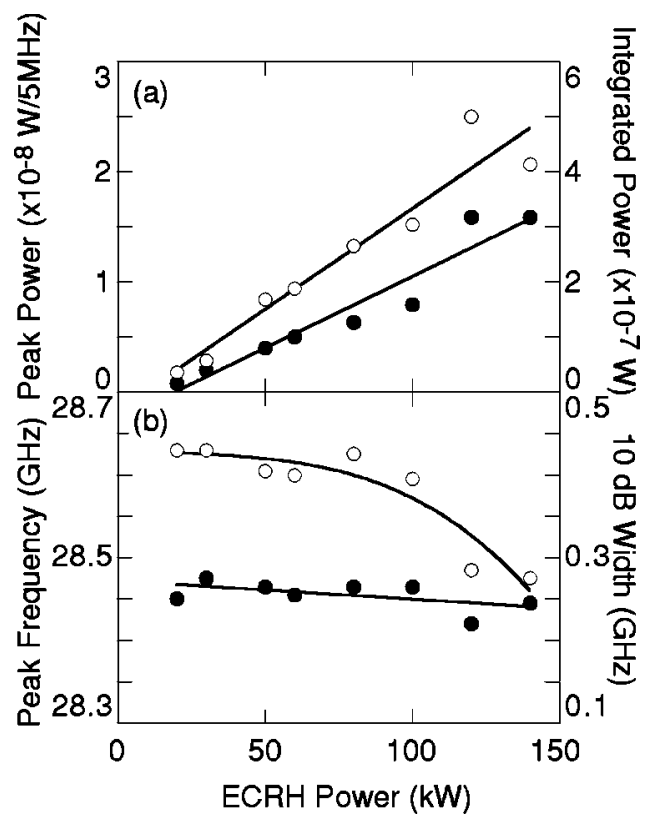

FIG. 6. Characteristic parameters of the narrow-band radiation are plotted against the plug ECRH power. (a) The peak power (closed circles) and the integrated power (open circles). (b) The peak frequency (closed circles) and the $10 \mathrm{~dB}$ width (open circles).

$\mathrm{GHz}$ examined. We interpret that this component corresponds to bulk electrons passing through the whole space of confinement.

The new observation we emphasize in this article is the narrow component rising in the spectrum between 28 and 29 GHz. The characteristic features are the following.

(1) The frequency of the highest intensity $f_{\text {peak }}$ lies slightly above the heating frequency $f_{H}$ with a clear separation of $0.1-0.5 \mathrm{GHz}$.

(2) The bandwidth $\Delta f_{\text {peak }}$ of the emission is narrow $\Delta f_{\text {peak }} / f_{H} \ll 1$ but much broader than the width of the heating wave $\Delta f_{H}<0.01 \mathrm{GHz}$.

(3) The intensity of the spectrum peak $P_{\text {peak }}$ grows with increasing power of the heating wave, but $f_{\text {peak }}$ stays around $28.4 \mathrm{GHz}$.

(4) The bandwidth $\Delta f_{\text {peak }}$ decreases with the heating power, namely the high frequency tail of the spectrum shrinks while the lower cutoff $(\leqslant 28.3 \mathrm{GHz})$ does not change appreciably.

These characteristics in the frequency spectrum are summarized quantitatively in Fig. 6. Figure 6(a) shows the peak power level $P_{\text {peak }}$ (closed circles) of this component and the frequency-integrated power (open circles) as functions of the ECRH power. The integration is made from 28.1 to 29.5 GHz. It is observed that the both power levels increase almost linearly with the heating power. So far, we have not found any threshold value in the heating power for the appearance of the narrow component. Figure $6(\mathrm{~b})$ plots the frequency $f_{\text {peak }}$ of the peak power (closed circles) and the $10 \mathrm{~dB}$ width $\Delta f_{\text {peak }}$ of this component (open circles) as functions of the ECRH power. The $10 \mathrm{~dB}$ width is about $0.4 \mathrm{GHz}$ and decreases with the increasing power of the ECRH. The peak 


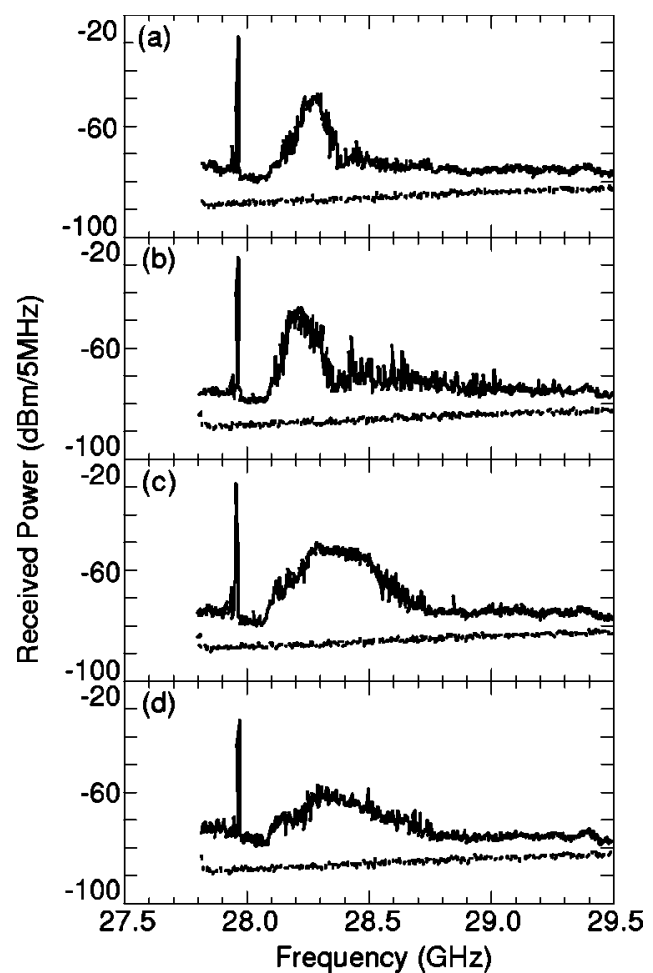

FIG. 7. Radiation spectra associated with different aiming angles of the heating beam. $\phi=3.5$ (a), 1.5 (b), -0.5 (c), -2.5 (d) deg.

frequency remains around $28.4 \mathrm{GHz}$ indicating a hint of a slight down-shift with increasing ECRH power.

We examine the contribution of the location of the resonant absorption to the radiation spectrum by changing the beam direction with the rotatable reflector. The angle $\phi$ of the reflector is fixed at $1.5 \mathrm{deg}$ in the standard operation. A positive increment of $1 \mathrm{deg}$ results in the upward shift of the power deposition by $8 \mathrm{~mm}$ on the $1 \mathrm{~T}$ surface. Figure 7 shows the frequency spectra observed at different angles of the reflector such as $\phi=3.5$ (a), 1.5 (b), -0.5 (c), and -2.5 (d) deg. In each plate, the plug ECRH is kept at 100 $\mathrm{kW}$. It is observed that the width of the narrow component increases for a decreasing angle of the reflector.

These observations of the frequency spectra are summarized in Fig. 8. Figure 8(a) shows the peak power level (closed circles) of the narrow component and the frequencyintegrated power (open circles) as functions of $\phi$. The integration is made from 28.1 to $29.5 \mathrm{GHz}$. Figure 8(b) plots the frequency of the peak power (closed circles) and the $10 \mathrm{~dB}$ width of this component (open circles) as functions of $\phi$. Both power levels are maximized at $\phi=1.5 \mathrm{deg}$. The peak frequency shifts upward on either side of $\phi=1.5 \mathrm{deg}$. The power efficiencies for electron heating and the potential formation are maximized at $\phi=1.5 \mathrm{deg}$ if we judge from the diagnostics of the plasma parameters and the potential differences along the magnetic axis. Thus, it is noticed that the appearance of the enhanced narrow-band spectra is pertinent to the optimized operation of the tandem mirror plasma in terms of the potential formation by ECRH.

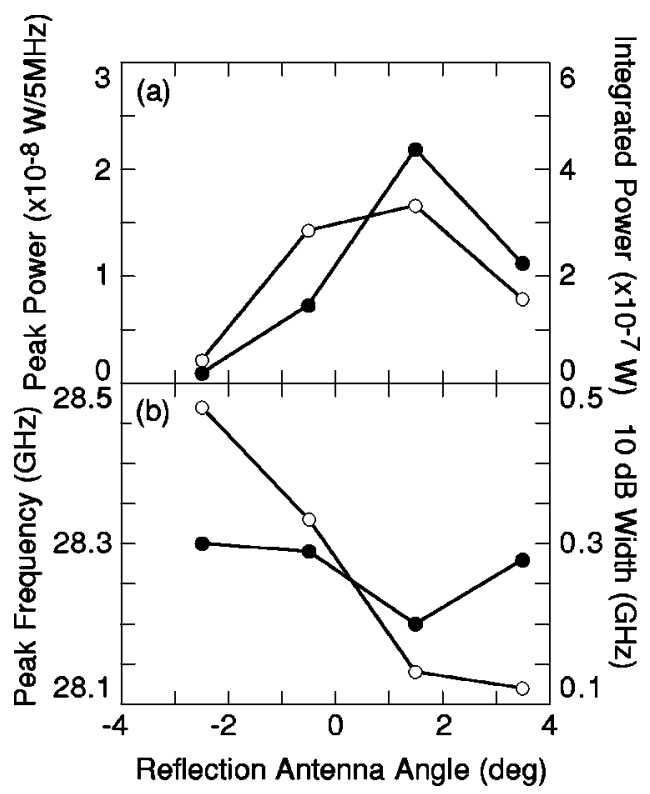

FIG. 8. Characteristic parameters of the narrow component are plotted against $\phi$. (a) The peak power (closed circles) and the integrated power (open circles). (b) The peak frequency (closed circles) and the $10 \mathrm{~dB}$ width (open circles).

\section{Emission in the second harmonic range}

If the strong narrow-band wave is generated by a process in which wave-wave coupling is involved, there is a possibility that nonlinearly coupled oscillations may generate an electromagnetic wave at the second harmonic range with a similarly peaked spectrum. To test this possibility, we examine the frequency spectrum in the second harmonic range. An example of the observed spectra is shown in Fig. 9. When the plug ECRH is applied at $100 \mathrm{~kW}$, the radiation power increases by several $\mathrm{dB}$ over a wide frequency range. This observation assures that we have sufficient sensitivity with our detection system to indicate the presence of waves if they exist. The sharp peak observed near $56 \mathrm{GHz}$ is not responsible for the emission mechanism but it is attributed to the heating wave that is frequency-doubled by nonlinear elements in the detection circuit.

Furthermore, once the signal level in the range 56-58 GHz goes up as in Fig. 9, it does not drop for more than 10 msec after the turn-off of the ECRH. The long lifetime of the emission is comparable with the long decay times of the

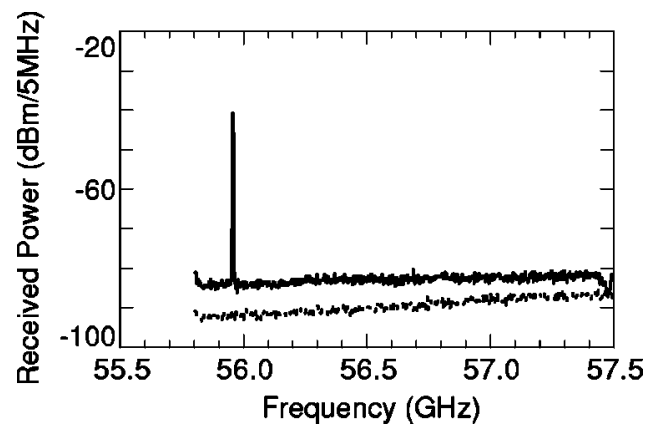

FIG. 9. Spectra in the second harmonic range. The dots represent the system noise level. 


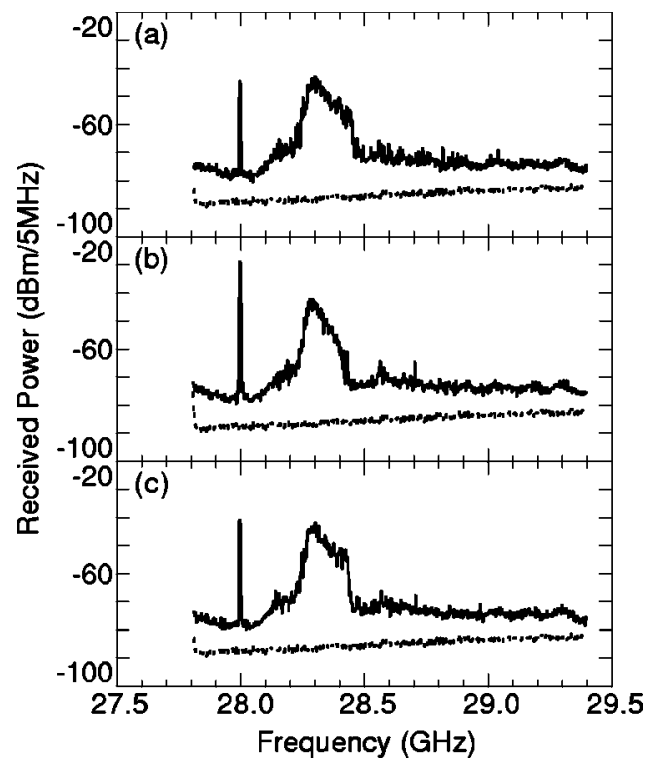

FIG. 10. Radiation spectra collected at different locations of the receiver antenna, $y=0.275$ (a), 0.523 (b), 0.822 (c) $\mathrm{m}$.

local diamagnetism and $\mathrm{x}$-ray emissions, so that the broad spectrum in the $56 \mathrm{GHz}$ range is attributed to incoherent cyclotron emissions from mirror-trapped hot electrons with energies $\geqslant 10 \mathrm{keV} .{ }^{14,17}$ The lack of correspondence between the two frequency bands in terms of the spectral shape and the time to be observed indicates that nonlinear wave-wave interactions in the high frequency range are not responsible for the generation of the strong narrow-band radiation near the fundamental resonance.

\section{Propagation of radiation}

If a resonance or a cut-off layer exists in the paths of the radiation propagating toward the detector antenna, the observed spectrum is different from that generated in the plasma. Namely, it is not self-evident that the narrow component is not produced as a result of a frequency-selective transparency of the plasma. As an experimental test, though quite limited, we examine frequency spectra of the radiation collected at different locations along different paths of the waves. The results are shown in Fig. 10 obtained with the plug ECRH power of $140 \mathrm{~kW}$. In each panel, the receiver antenna is placed at $y=0.275,0.523$ and $0.822 \mathrm{~m}$, downward. Since the frequency spectra do not change appreciably with $y$, we may consider that the observed spectra are not the results of the filtering along the path of the wave propagation.

This experiment-based statement is supported by a raytracing analysis ${ }^{18}$ as shown in Fig. 11. The idea is to examine the possibility that waves in a broad band of frequency around $28 \mathrm{GHz}$ are blocked to propagate out to the receiver region. For simplicity, we show the case that each wave starts from its resonant point on the magnetic axis. Here we employ geometrical optics in warm electrons of biMaxwellian distribution with temperatures $T_{\perp} / T_{\|}=500 / 100$ in $\mathrm{eV}$ and the density distribution fitting to the actual plasma. Figure 11 shows paths of different frequency components,

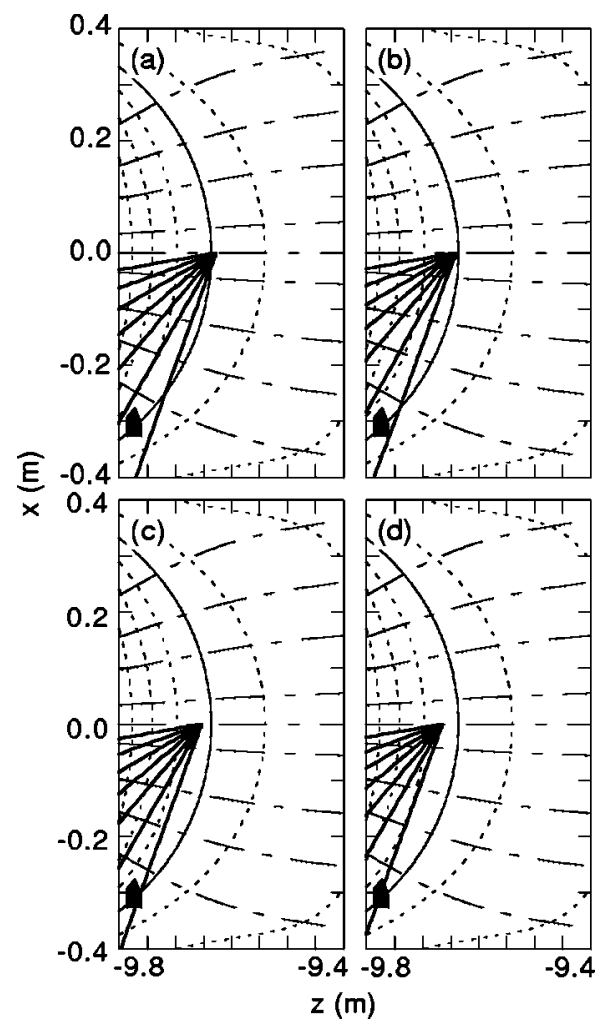

FIG. 11. Paths of different frequency components are shown with thick solid lines. The frequencies are (a) 27, (b) 28, (c) 29, (d) $30 \mathrm{GHz}$. Each frequency-component starts from the resonance point on the magnetic axis. The dash-dotted curves represent the magnetic field lines. The dotted lines shows the mod-B surfaces with equal separation of $0.1 \mathrm{~T}$, while the solid curve denotes the surface of $B=1 \mathrm{~T}$. The dark box stands for the receiver antenna.

(a) 27, (b) 28, (c) 29 and (d) $30 \mathrm{GHz}$. It is confirmed that every component selected from a wide frequency range can reach the receiver antenna without severe damping if it is generated. This results, combined with the observation in Fig. 10, indicates that the narrow-band emissions are not the results of the frequency selective propagation of waves generated in a wide band.

\section{DISCUSSION}

We discuss the physical mechanism of the generation of the strong radiation in the narrow band. The main issue includes the power level and the frequency width. We have already discarded the possibility that the narrow band is attributed to the filtered propagation of waves with broader spectra. The spectrum analyses at the second harmonic range have shown that nonlinear self-interaction of the heating wave is also unlikely. Scattering of the heating wave by electrons could produce a narrow-band spectrum. It may be interpreted in terms of radiation associated with acceleration by the heating wave field. This process is also less likely than electron cyclotron emission because the electric acceleration $\left(e / m_{e}\right) E$ by the wave field $E$ is more than two orders of magnitude smaller than the magnetic acceleration $\left(e / m_{e}\right) v \times B$ even with the maximum heating power. The only conceivable process that we do not have direct means to test is the collective scattering of a heating wave by fluctua- 


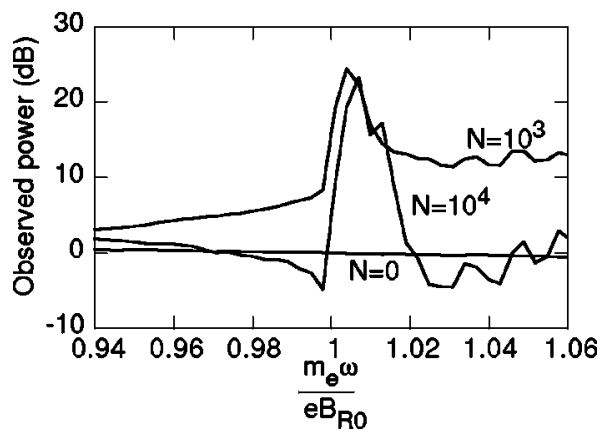

FIG. 12. Calculated frequency spectra of incoherent cyclotron emission from heated electrons. The electron velocity distribution is calculated by applying the heating response function (Refs. 19 and 20) to the actual experimental configuration. The number attached to each spectrum denotes the maximum number that electrons experience resonance heating while they are trapped in the mirror field. The calculation includes the effect that electrons disappear after driven into the loss-cone. This figure is taken from Ref. 21.

tions with frequency ranging below $0.5 \mathrm{GHz}$. So far, we do not have observations that suggest the existence of such fluctuations. Therefore, we proceed with our discussion in terms of electron cyclotron radiation.

The observed narrow band requires that the enhanced electron cyclotron emission should be highly localized in terms of the magnetic field strength. The axial length $\Delta z$ of the distribution of relevant electrons may be evaluated as

$$
\Delta z=\frac{B_{0}}{\left|d B_{0} / d z\right|} \frac{\Delta f_{\text {peak }}}{f_{H}}
$$

Because $B_{0} /\left|d B_{0} / d z\right| \approx 0.45 \mathrm{~m}$, and $\Delta f_{\text {peak }} / f_{H} \approx 0.4 / 28$, we obtain a small value of $\Delta z=6.4 \times 10^{-3} \mathrm{~m}$. The number of electrons $N$ responsible for the narrow-band emission is estimated to be

$$
N=\pi a^{2} \Delta z \times n_{e} \approx 2 \times 10^{13}
$$

with the plasma radius $a=0.1 \mathrm{~m}$ and the electron density $n_{e}=10^{17} \mathrm{~m}^{-3}$. This type of localization of heated electrons is actually obtained by numerical analyses of the electron orbits in the presence of a monochromatic electron cyclotron wave in a magnetic beach by using the heating response function. ${ }^{19,20}$ Realistic experimental parameters are used in the calculation, and the velocity distribution is found to become highly anisotropic when heated electrons are trapped in the magnetic mirror and cross the resonance layer many times. The frequency spectrum calculated as a superposition of individual ECE from those electrons are taken from Ref. 21 and shown in Fig. 12. The good correspondence in shape between the observed spectra and the calculation indicates that ECE is the most probable mechanism of the strong radiation.

The next issue to be discussed is about the observed power of the radiation. If we neglect the thermal spread along the magnetic field and only take $T_{\perp}$ into account, the emissivity $j(\omega, \theta)$ near the electron cyclotron resonance is calculated as, ${ }^{3}$

$$
j(\omega, \theta)=\frac{e^{2} \omega^{2} n_{e} T_{\perp}}{32 \pi^{2} \epsilon_{0} c^{3} m_{e}}\left(1+\cos ^{2} \theta\right) \delta\left(\omega-\omega_{c}\right) .
$$

Here $\theta$ is the direction of the propagation with respect to the magnetic field. From this expression the power density of the radiation at the receiving antenna may be evaluated as

$$
p_{t}=\frac{\gamma N}{4 \pi R^{2}} \frac{e^{2} \omega_{c}^{2} T_{\perp}}{32 \pi^{2} \epsilon_{0} c^{3} m_{e}} \approx 3.0 \times 10^{-9} \frac{\gamma T_{\perp}}{R^{2}},
$$

where $R$ is the distance to the receiver antenna, and $\gamma$ is a geometrical factor ranging from 1 to 10 . Introducing $T_{\perp}$ $=10^{3} \mathrm{eV}, R=0.3 \mathrm{~m}$ and $N$ in Eq. (3), we obtain $p_{t}=3$ $\times 10^{-5} \gamma \mathrm{W} / \mathrm{m}^{2}$.

The power density $p_{r}$ of the narrow-band radiation at the receiver antenna may be evaluated from the power level $P_{\text {peak }}$ and the width $\Delta f_{\text {peak }}$ which are read on the spectrum analyzer as

$$
p_{r}=\frac{1}{A_{e}} \frac{P_{\text {peak }}}{\eta_{f}} \frac{\Delta f_{\text {peak }}}{5 \mathrm{MHz}},
$$

where the transmission efficiency $\eta_{f}=-20 \mathrm{~dB}$ and the effective area of the receiver antenna $A_{e}=6 \times 10^{-5} \mathrm{~m}^{2}$ are to be used. From a typical parameter $P_{\text {peak }} \Delta f_{\text {peak }} / 5 \mathrm{MHz}=3$ $\times 10^{-7} \mathrm{~W}$ as given in Fig. 8, we obtain $p_{r}=0.5 \mathrm{~W} / \mathrm{m}^{2}$.

Though the anisotropic velocity distribution of heated electrons successfully explains the shape of the observed frequency spectra, the observed power is three orders of magnitude higher than the theoretical expectation for $N$ as estimated in Eq. (3). To compensate for the large difference, we finally introduce an additional process that is compatible with the processes discussed above. It is well known that electrons are bunched in the velocity space when interacting with a monochromatic cyclotron wave and spatial bunching may appear after a second interaction. ${ }^{22}$ In the intense electric field $\left(E=10^{4}-10^{5} \mathrm{~V} / \mathrm{m}\right)$ of the heating wave, one can expect a strong bunching of electrons that are resonantly heated and mirror-reflected.

If $N_{b}$ electrons are spatially bunched into clusters and gyrate synchronously, the emissivity per cluster is proportional to $\left(N_{b} e\right)^{2}$. Because the maximum number of clusters that can form from $N$ electrons is $N / N_{b}$, the amount of emission could be enhanced by up to a factor $N_{b}$. To make the model meet the observation, we would need $N_{b}=N_{b 0} \approx 10^{3}$ for the fully bunched case. This requirement can be relaxed so that $\beta N$ electrons are to be bunched while $(1-\beta) N$ electrons remain randomly distributed, where 0 $<\beta<1$. To generate the same amount of emission from $\beta N$ electrons, we need $\beta^{2} N / N_{b 0}$ clusters of $N_{b 0} / \beta$ electrons. Therefore, even the partial bunching with $\beta=0.1$ can account for the observed power level if the number of electrons per cluster is increased from $10^{3}$ to $10^{4}$. Such a state of electron bunching can never be unrealistic because $\beta$ roughly corresponds to the modulation level of the local electron density near the resonance layer. Though there is no direct evidence of the spatial bunching, we do expect that only this process can account for the observed enhancement of emission with realistic requirements for plasma parameters but without any contradictory observations. 


\section{v. CONCLUSION}

In conclusion, under the magnetic beach heating of collisionless electrons with a monochromatic cyclotron wave, we have observed an enhanced radiation spectrum that consists of two components. One is a broad component that is attributed to incoherent cyclotron emissions of heated electrons. The other much stronger component is limited in a narrow range above the heating frequency. This component is generated by collective cyclotron emission from electrons locally trapped in the mirror field and bunched by the strong monochromatic heating wave.

\section{ACKNOWLEDGMENTS}

The authors appreciate the collaborative work with members of the GAMMA 10 group. This work was supported by the Grant-in-Aid program of Ministry of Education, Science, Sports and Culture of Japan.

${ }^{1}$ G. Bekefi, Radiation Processes in Plasmas (Wiley, New York, 1966), pp. 177-213.

${ }^{2}$ M. A. Heald and C. B. Wharton, Plasma Diagnostics with Microwaves (Wiley, New York, 1966), pp. 272-280.

${ }^{3}$ I. H. Hutchinson, Principles of Plasma Diagnostics (Cambridge University Press, New York, 1987), pp. 135-160.

${ }^{4}$ M. Bornatici, R. Cano, O. DeBarbieri and F. Engelmann, Nucl. Fusion 23, 1153 (1983).
${ }^{5}$ T. H. Stix, Waves in Plasma (American Institute of Physics, Woodbury, 1992), Chaps. 11-12.

${ }^{6}$ R. F. Ellis, G. D. Tsakiris, and D. A. Boyd, Phys. Fluids 26, 1528 (1983).

${ }^{7}$ T. Kariya, T. Saito, Y. Kiwamoto, T. Cho, H. Sugawara, T. Kondoh, A. Mase, A. Itakura, and S. Miyoshi, Phys. Fluids 30, 1815 (1988).

${ }^{8}$ Y. Kiwamoto, T. Saito, I. Katanuma, K. Kurihara, and S. Miyoshi, J. Phys. Soc. Jpn. 58, 2619 (1989).

${ }^{9}$ T. Saito, I. Katanuma, Y. Kiwamoto, T. Kariya, and S. Miyoshi, Phys. Rev. Lett. 59, 2748 (1987).

${ }^{10}$ T. Cho, M. Hirata, E. Takahashi et al., Phys. Rev. Lett. 64, 1373 (1990).

${ }^{11}$ R. H. Cohen, Phys. Fluids 26, 2774 (1983).

${ }^{12}$ M. Inutake, T. Cho, M. Ichimura et al., Phys. Rev. Lett. 55, 939 (1985).

${ }^{13}$ T. Tamano, Phys. Plasmas 2, 2321 (1995).

${ }^{14}$ T. Saito, Y. Kiwamoto, T. Kariya, K. Kurihara, I. Katanuma, T. Cho, and S. Miyoshi, Nucl. Fusion 30, 1533 (1990).

${ }^{15}$ K. Kurihara, Y. Kiwamoto, T. Saito, K. Yatsu, and S. Miyoshi, J. Phys. Soc. Jpn. 61, 3153 (1992)

${ }^{16}$ S. N. Vlasov, G. M. Zhislin, I. M. Orlova, M. I. Petelin, and G. G. Rogacheva, Izv. Vyssh. Uchebn. Zaved., Radiofiz. 12, 1236 (1969).

${ }^{17}$ Y. Kiwamoto, T. Cho, T. Saito et al., Phys. Fluids 29, 2781 (1986).

${ }^{18}$ M. Porkolab, L. Friedland, and I. B. Bernstein, Nucl. Fusion 21, 1643 (1981).

${ }^{19}$ Y. Kiwamoto, T. Saito, Y. Tatematsu, I. Katanuma, Y. Yoshimura, and T. Tamano, Phys. Plasmas 1, 834 (1994).

${ }^{20}$ Y. Tatematsu, Y. Kiwamoto, T. Saito, I. Katanuma, Y. Yoshimura, and T. Tamano, Phys. Plasmas 3, 3318 (1996).

${ }^{21}$ Y. Tatematsu, T. Saito, Y. Kiwamoto, K. Ito et al., to be published in Fusion Eng. Design.

${ }^{22}$ R. W. Gould, Am. J. Phys. 37, 585 (1969). 